\title{
Molecular analysis of the replication program in unicellular model organisms
}

\author{
M. K. Raghuraman • Bonita J. Brewer
}

Published online: 10 December 2009

(C) Springer Science+Business Media B.V. 2009

\begin{abstract}
Eukaryotes have long been reported to show temporal programs of replication, different portions of the genome being replicated at different times in S phase, with the added possibility of developmentally regulated changes in this pattern depending on species and cell type. Unicellular model organisms, primarily the budding yeast Saccharomyces cerevisiae, have been central to our current understanding of the mechanisms underlying the regulation of replication origins and the temporal program of replication in particular. But what exactly is a temporal program of replication, and how might it arise? In this article, we explore this question, drawing again on the wealth of experimental information in unicellular model organisms.
\end{abstract}

Keywords origin · replication · timing ·

temporal program $\cdot$ yeast

Abbreviations used
$\begin{array}{ll}\text { ARS } & \text { Autonomous replication sequence } \\ \text { BAC } & \text { Bacterial artificial chromosome } \\ \text { BrdU } & \text { Bromodeoxyuridine } \\ \text { ChIP } & \text { Chromatin immunoprecipitation }\end{array}$

Responsible Editors: Marie-Noëlle Prioleau and Dean Jackson.

M. K. Raghuraman $(\bowtie) \cdot$ B. J. Brewer

Department of Genome Sciences,

University of Washington,

Box 355065, Seattle, WA 98133, USA

e-mail: raghu@u.washington.edu
HU Hydroxyurea

ORC Origin recognition complex

pre-RC Prereplicative complex

ssDNA Single-stranded DNA

\section{Introduction}

Chromosomal DNA replication is the act that gives meaning to cell division. Replicating the genome is a task of immense complexity and yet must be managed with superb fidelity to ensure genomic integrity. On each eukaryotic chromosome, DNA synthesis begins at hundreds or thousands of sites, the origins of replication. It has long been observed that eukaryotic genomes show a temporally staggered pattern of replication, with different parts of the genome being replicated on average at different times in $\mathrm{S}$ phase, and that, at least in metazoans, early replication is correlated with transcriptional activity or the potential for transcriptional activity (Schübeler et al. 2002; Woodfine et al. 2004; Donaldson 2005). Naturally, there is strong motivation to understand the mechanisms regulating origin specification and replication timing. Since organisms as diverse as the budding yeast $S$. cerevisiae and mammals such as humans show temporal domains of replication and the molecular mechanisms underlying the regulation of origin initiation or "firing" are strongly conserved across evolution (see Bell and Dutta 2002 for a review), the hope has been that experimentally amenable 
unicellular eukaryotes such as $S$. cerevisiae and the fission yeast Schizosaccharomyces pombe can serve as surrogates to understand the replication dynamics of the more complex metazoan genomes. For the most part, this approach has been wildly successful: much of what we know about the molecular details of origin initiation and its regulation were first worked out in budding yeast (Bell and Dutta 2002).

Nevertheless, while the mechanisms controlling origin activation are increasingly being elucidated, the details of the mechanisms underlying the observed temporal pattern of replication remain obscure. Furthermore, increased scrutiny in recent years has raised the possibility that mechanisms may vary across species: that the program may be more stochastic in some species (e.g., S. pombe) than in others (e.g., S. cerevisiae).

In this article, we explore some mechanistic models for how temporal patterns of replicationthe "temporal program"-may be established and discuss some avenues of investigation for the future. Because conclusions we can draw from experimental work are so dependent on a clear understanding of the methodology, we begin with a brief survey of some of the techniques in use and projected for the near future, summarizing along the way some of what we have learned and discussing some recent new ideas on how the temporal program may be established. Towards the end, we take a brief look at the promise and the contribution of computational approaches to understanding replication and speculate on the evolution of the replication program. Our focus here is on simple unicellular model systems (primarily, S. cerevisiae) with occasional discussion of the implications for higher eukaryotes.

But first - what exactly is a temporal program of origin activation? The term can mean different things to different people. The idea of a temporal program goes back to the original observation by Taylor (1960) that $\left[{ }^{3} \mathrm{H}\right]$-thymidine given as a pulse to cultured Chinese hamster cells was incorporated into different portions of the genome at different times in S phase - an observation which, in the words of the author, "led to the hypothesis that various chromosomes or parts of chromosomes have a genetically controlled sequence in duplication which may have some functional significance." As techniques improved and studies on $S$. cerevisiae gained prominence, the concept of a temporal program came to include the idea of a temporally regulated series of origin activations (Fangman and Brewer 1992). The word "program" has connotations of a rigid, predetermined series of events - which, as discussed below, is not believed to be an appropriate description of origin initiations - but the term nevertheless is in widespread use. In this article, we use the term simply in the empirical sense: that on average, in bulk populations of cells, there is an observed propensity for different portions of the genome to be replicated at different times in $\mathrm{S}$ phase.

\section{Investigating the topography of replication}

What is the evidence that origin activations are staggered in S phase? How does one know when an origin fires? To obtain this information, it helps to know where origins are located (or what their nucleotide sequences are), which then allows us to map their replication times. In $S$. cerevisiae, potential origins can be identified using a plasmid maintenance assay-i.e., by finding genomic DNA sequences that allow maintenance of plasmids that otherwise would be incapable of autonomous replication (Stinchcomb et al. 1980). A sequence thus identified as capable of supporting replication of a plasmid is termed an autonomous replication sequence (ARS).

The firing time of a potential origin so identified historically was inferred from two assays: a twodimensional (2-D) agarose gel electrophoresis assay (Brewer and Fangman 1987) to confirm that the ARS actually functions as an origin in its native chromosomal context in most cells and a replication timing assay to monitor the kinetics of its replication. The timing assay that is often used is a variation of the Meselson-Stahl dense isotope transfer method (Meselson and Stahl 1958; McCarroll and Fangman 1988) wherein cells are grown for many population doublings in isotopically dense culture medium so that their DNA is uniformly labeled with the dense isotope on both strands and is thus "heavy-heavy" (HH). The cells are synchronized at or just prior to the G1/S phase boundary and allowed to enter $\mathrm{S}$ phase in isotopically normal (light) medium so that newly replicated DNA is hybrid in density (heavylight, "HL"). Samples are collected during S phase and replicated (HL) genomic restriction fragments are separated from unreplicated (HH) fragments by centrifugation in cesium chloride. Fractionated gradients are 
hybridized on a slot blot to specific sequences of interest to determine the kinetics of their replication. Despite the best efforts at cell synchronization, these kinetic curves show a sigmoidal shape rather than a sharp step function (as would be expected if all cells in the population replicated that particular sequence at the same time), indicative of some asynchrony in the population - cellto-cell variation in either their rate of progress through the cell cycle or in the timing of replication of different parts of the genome. To facilitate comparisons between different sequences, the replication time of each sequence is represented by its $t_{\text {rep }}$, its time of halfmaximal replication.

From the combination of these two assays (2-D gels to confirm activity and density transfer to monitor the kinetics of replication), the activation kinetics of the origin can be deduced and has provided evidence in yeast of staggered activation times of origins that reflect a population average (Fig. 1). [An alternative method that has also been used to time a fragment is to do 2-D gel analysis of S phase samples (e.g., Yamashita et al. 1997). This method gives an estimate of the approximate time of origin firing but is relatively laborious and does not readily lend itself either to quantitative measurements or to genome-wide surveys.]

This overall approach has been extremely successful at identifying and testing individual origins in $S$. cerevisiae, demonstrating that origins in this yeast show a staggered pattern of activation in $\mathrm{S}$ phase and that, in general, centromeric regions replicate early in $\mathrm{S}$ phase while telomeric regions replicate late (McCarroll and Fangman 1988; Ferguson et al. 1991). By the turn

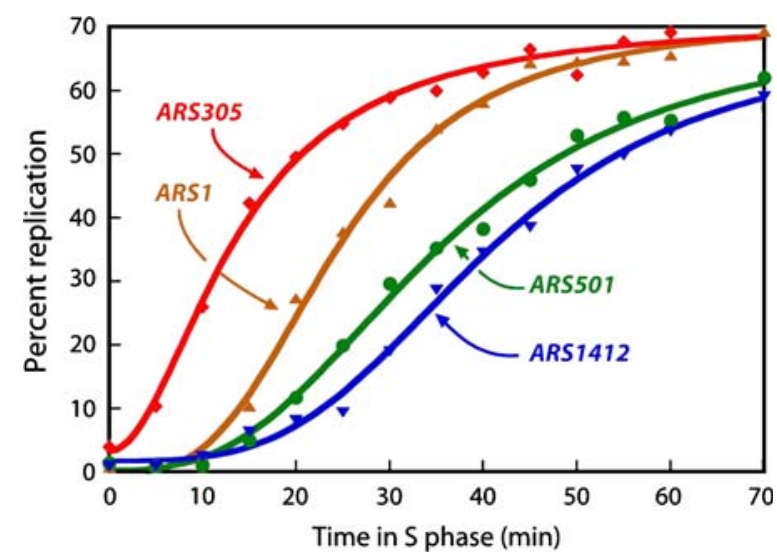

Fig. 1 The temporal program of replication illustrated by the kinetics of replication of four efficient origins in $S$. cerevisiae of the century, these techniques had been used to obtain low-resolution replication maps of small yeast chromosomes, giving origin locations and their inferred times of activation (Friedman et al. 1997; Yamashita et al. 1997; van Brabant et al. 1998). Furthermore, the ARS assay enabled genetic dissection of the sequences needed for origin function, revealing that $S$. cerevisiae origins consist of a 100-200 bp sequence with a modular structure: an "A" domain containing an essential, 11-bp ARS consensus sequence (ACS), along with "B" and "C" elements, which vary in sequence between different ARSs (Marahrens and Stillman 1992; Newlon and Theis 1993). The ACS and a subdomain of the $\mathrm{B}$ element together act as the binding site for the origin recognition complex (ORC; Bell and Stillman 1992; Diffley and Cocker 1992), a heterohexamer that acts as a "landing pad" to recruit the proteins that make up the prereplicative complex (pre-RC, reviewed by Zannis-Hadjopoulos et al. 2004)-i.e., an origin biochemically "licensed" to fire as had been proposed previously (Rao and Johnson 1970; Blow and Laskey 1988). This picture of $S$. cerevisiae origins has largely held up over the years, with a few refinements, e.g., expansion of the ACS to a 17-bp sequence (Theis and Newlon 1997) and the realization that some origins have a compound structure with multiple ACSs (Theis and Newlon 2001).

This model for investigating replication patterns has had some mixed success when applied to other species. For example, ARSs in $S$. pombe have been found to be 600-1,500 bp long (Clyne and Kelly 1995), much larger than in $S$. cerevisiae and with no specific consensus sequence beyond an A/T-rich sequence, while in Kluyveromyces lactis, ARS elements appear to contain a $\sim 50$-bp essential consensus sequence (Irene et al. 2004). ARS assays have not proven to be informative in human cell systems, however, as any human genomic DNA insert, if large enough ( $\geq 12 \mathrm{~kb})$, was found to be capable of promoting plasmid retention (Heinzel et al. 1991). Due to these other species generally being either less developed as model organisms or less tractable than $S$. cerevisiae, replication timing analysis has not been as straightforward. In S. pombe, for example, timing analysis was attempted based on 2-D gel electrophoresis of $\mathrm{S}$ phase samples (Kim and Huberman 2001). In mammalian systems, various methods were applied - e.g., in situ hybridization with fluorescent probes to allow timing of the expected doubling in the number of hybridizing foci 
concomitant with the replication of the target sequence and labeling of sites of replication with analogs such as bromodeoxyuridine (BrdU) followed by cell sorting to "stage" the cells in the population according to their progress through S phase (Hansen et al. 1995; Gartler et al. 1999).

Within the last decade, however, new techniques and modifications of old ones have galvanized the field, promising high-resolution, whole-genome glimpses into the dynamics of replication. As before, studies in $S$. cerevisiae have led the way and have produced the most detailed information, but considerable progress is being made with other species also. In $S$. cerevisiae, these techniques together have led to the identification of virtually all the origins and additionally have elucidated the general order of replication within the genome. Some of the methods used to survey replication dynamics across the genome are summarized below.

- Chromosomal replication profiles (Fig. 2) encapsulating information on the locations of active origins (peaks in the plots), termination zones (valleys), relative times of replication of loci across the chromosome (on the $Y$-axis), and relative rates of fork migration (slopes of the lines connecting peaks and valleys) can be obtained by a modification of the density transfer method or simply by measuring relative copy number of different portions of the genome in S-phase cells. In the density transfer method, the principle is unchanged, but instead of slot-blot hybridization to monitor the replication kinetics of a single genomic restriction fragment, $\mathrm{HH}$ (unreplicated) and HL (replicated) DNA from each S-phase sample are labeled separately and hybridized to DNA microarrays, giving a snapshot of the extent of genomic DNA replication at that time in $\mathrm{S}$ phase (Raghuraman et al. 2001; Alvino et al. 2007). Copy number measurement is a simple concept: as a cell population undergoes a synchronous $\mathrm{S}$ phase, the progressive increase in copy number (from $1 \mathrm{C}$ to 2C) of each portion of the genome can be monitored by quantitative hybridization (e.g., Yabuki et al. 2002). In principle, the same goal could be achieved by newer sequencing technologies, although to date microarray hybridization has been the norm (but see Desprat et al. 2009).

- ChIP-chip or ChIP-seq: chromatin immunoprecipitation with antibodies against particular pro- teins of interest, e.g., ORC subunits, followed by microarray hybridization or sequencing of the DNA that was coimmunoprecipitated reveals locations of ORC binding and, presumably, locations that could form pre-RCs and, ultimately, serve as origins (Wyrick et al. 2001; Xu et al. 2006). Because not all pre-RCs actually proceed to initiate DNA synthesis (Santocanale and Diffley 1996), the ChIP method identifies potential origins, of which actual origins would be a subset (Fig. 2).

- Mapping sites of single-stranded DNA (ssDNA) in cells entering $\mathrm{S}$ phase in the presence of the replication-impeding drug hydroxyurea (HU) detects ssDNA at replication bubbles and thereby marks origins that fire early in S (Feng et al. 2006). The presence of $\mathrm{HU}$ causes a slowdown of $\mathrm{S}$ phase, substantially delaying origin firing (Alvino et al. 2007). Therefore, in wild-type cells, transient accumulation of ssDNA is seen at mostly earlyfiring origins. In a rad53 checkpoint mutant, this delay in firing is abrogated, and sites of ssDNA reveal essentially the full complement of active origins in the genome (Feng et al. 2006; Feng et al. 2007; Fig. 2).

- Incorporation of halogenated analogs such as bromodeoxyuridine labels sites of ongoing DNA synthesis and can identify sites where DNA synthesis is initiated. This approach is particularly intriguing, as it can then be followed up either with population-based studies (e.g., by hybridization of labeled DNA to microarrays to produce replication profiles as with density transfer experiments; Knott et al. 2009) or with single-molecule analysis of stretched DNA (e.g., Lengronne et al. 2001; Norio et al. 2005).

Many of these studies rely on synchronization of cell populations to allow their sampling as the population progresses through $\mathrm{S}$ phase. The use of synchronization regimens such as treatment or $S$. cerevisiae with mating pheromone or the use of conditional mutations in genes needed for onset of S phase has been thought to be benign treatments; nevertheless, with improvements in technology, origin mapping and replication timing studies increasingly are being done on asynchronous populations of cells (e.g., Lucas et al. 2007; Desprat et al. 2009). 


\section{a}

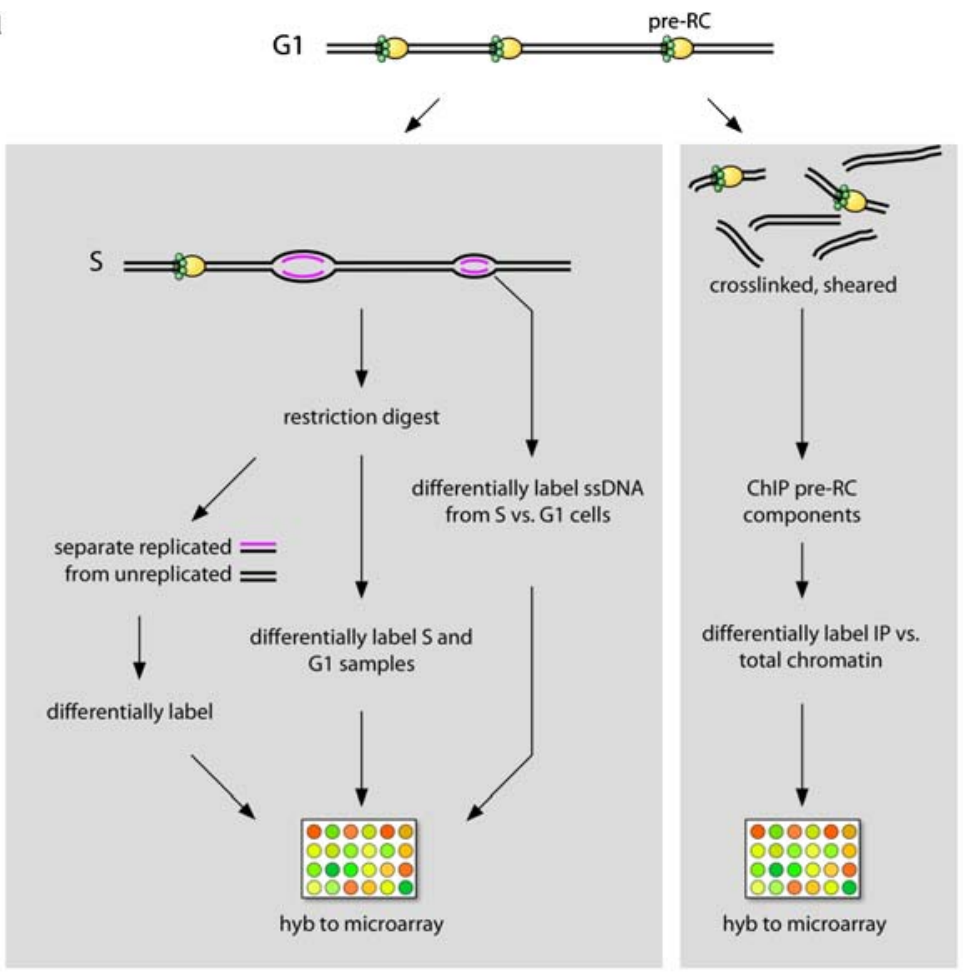

b

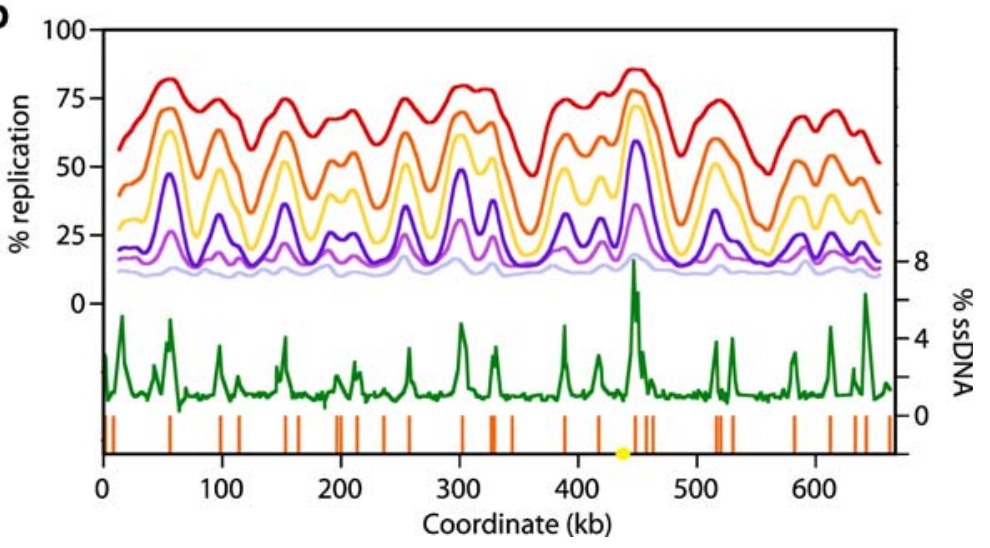

Fig. 2 Whole-genome replication profiling. a Cartoon illustrating popular profiling methods. Density transfer experiments to separate replicated from unreplicated DNA or BrdU labeling to specifically label and isolate newly synthesized DNA can be used to construct replication profiles - progressive snapshots of the extent of replication across the genome-revealing general features of replication such as origin locations and the kinetics of replication for different genomic loci. Similar results can be obtained by copy number measurements. More precise determination of origin locations can be obtained by mapping ssDNA accumulated during $\mathrm{S}$ phase, occurring in the presence of HU. Mapping ssDNA in rad53 checkpoint mutants reveals all active origins, while similar experiments in wild-type cells reveals origins that fire predominantly early in S phase. Potential origins can be identified by chromatin immunoprecipitation (ChIP) of origin-binding proteins such as ORC followed by microarray hybridization or sequencing of the immunoprecipitated DNA. b Replication profiles for S. cerevisiae chromosome XI obtained by density transfer analysis at progressive S-phase times (pale blue through red profiles), ssDNA mapping (green profile), and ChIP of ORC and $\mathrm{Mcm}$ proteins (revealing potential origins; vertical orange bars). Yellow dot on $x$-axis, location of centromere. Profiles based on data from Feng et al. (2006), Xu et al. (2006), McCune et al. (2008) 


\section{Origin efficiency and firing time}

Despite this wealth of information, key aspects of the replication program still remain unresolved, in part because most of these studies are done and data are collected on populations of cells. The observations and interpretations of the results therefore represent what the bulk of the cells are doing, and for the most part do not speak to what individual cells may be doing. Thus, a peak in a replication profile is evidence of origin initiation at that chromosomal location, but because the profile is generated from a population of cells, we generally cannot tell whether or not that origin fired in every cell in the population-i.e., the presence of an active origin can be detected by such means but not origin efficiency or firing success, the percentage of cells in the population that actually fired the origin (see Nomenclature). Origins have been shown to vary widely in efficiency, some firing in almost every cell cycle while others fire in $<10 \%$ of the cells (e.g., Friedman et al. 1997). Measuring origin efficiency is not straightforward. While 2-D gel electrophoresis can give a qualitative measure (or a semiquantitative measure, by measuring fork direction on either side of an origin using a modified version of the 2-D gel technique; Friedman and Brewer 1995) of origin efficiency, it does not lend itself to genome-wide analysis. A further consequence of not knowing the firing efficiency of an origin is that its $t_{\text {rep }}$ does not necessarily represent the time in $\mathrm{S}$ phase that it fires-the observed $t_{\text {rep }}$ is a composite value reflecting those cells in the population that actually fired the origin and those in which the origin was replicated passively by an incoming fork. An origin that replicates late in S phase could be doing so either because it fires efficiently late in $\mathrm{S}$ phase or because it fires early but is inefficient and therefore is replicated passively later in S phase in the majority of cells.

The upshot is that despite all our detailed genomewide knowledge of replication times, the actual (approximate) firing times of origins has been ascertained for only a small number of origins even in $S$. cerevisiae-based on 2-D gel analysis (e.g., Yamashita et al. 1997) or inferred for efficient origins from the progressive change in replication profiles seen in timed S-phase samples (McCune et al. 2008). Genome-wide origins have been classified broadly as early- or late-S firing based on whether or not they

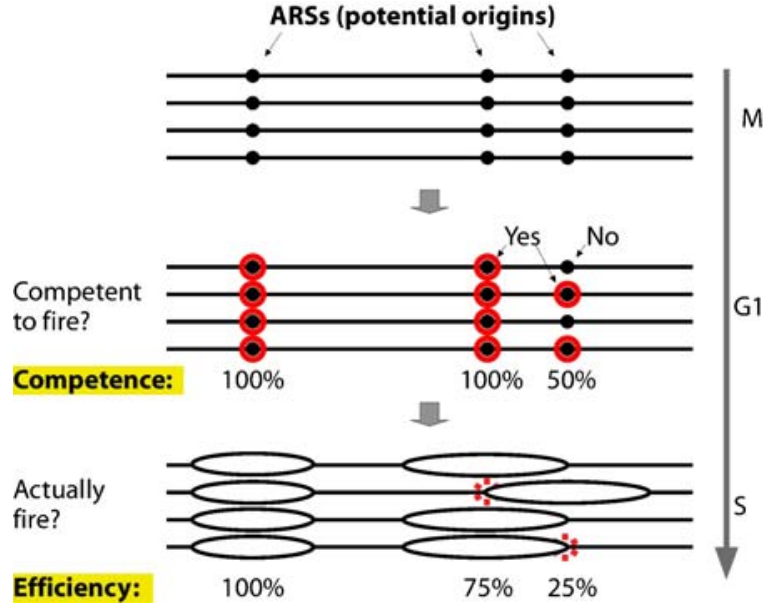

Nomenclature Origin efficiency: the percentage of cells in which an origin is observed to initiate DNA synthesis (or "fire") in any given S phase. Origin competence: the percentage of cells in which an origin is biochemically competent to fire. Firing probability or instantaneous firing probability: the probability that an origin will fire during a particular interval within S phase. Firing rate or spatiotemporal initiation density: the number of initiations occurring per unit of time per length in the genome. The relationship between competence and origin efficiency is illustrated in the diagram above (modified from McCune et al. 2008). Depicted are three ARSs in a four-cell population as it enters $\mathrm{S}$ phase. An ARS has the potential to be used as an origin. It may be $100 \%$ competent if it successfully becomes biochemically competent to fire (red ring) in every cell in the population, or it may show $<100 \%$ competence if it fails to achieve biochemical competence in some cells in the population. In those cells in which the origin failed to become competent, it would simply be replicated passively. The same fate may befall an origin that is competent but nevertheless is passively replicated by an incoming fork arriving at the origin before it has a chance to fire (broken red ring). Origin efficiency is the percentage of cells in which the origin actually fires

show evidence of firing in the presence of $\mathrm{HU}$ (Yabuki et al. 2002; Feng et al. 2006), which causes a $R A D 53$ checkpoint-mediated inhibition of unfired origins. Origins that, in wild-type cells, fail to fire after a brief exposure to $\mathrm{HU}$ therefore are equated with ones that normally fire late in S phase. However, to date, we do not have detailed genome-wide knowledge about the efficiency and firing times of origins in S. cerevisiae. In S. pombe, the amount of synthesis occurring in hydroxyurea-treated cells (as judged by increase in copy number) has been taken to be a measure of origin efficiency (Heichinger et al. 2006). The inferred efficiencies for 15 of the origins matched those measured using single-molecule anal- 
ysis (Patel et al. 2006). However, both studies relied on HU treatment; it remains to be seen how well these inferred efficiencies would match measurements made without HU treatment. These limitations notwithstanding, the general trend is clear and is also seen by 2-D gel analysis: most $S$. pombe origins show low efficiency compared to origins in $S$. cerevisiae (e.g., Segurado et al. 2003) although some origins do show fairly high efficiency (e.g., ori2004 in Okuno et al. 1997; ori2072, ori3043, and ori3044 in Segurado et al. 2003).

Why might an origin show $<100 \%$ efficiency? First, the observed efficiency of an origin depends on its competence - the probability that an origin biochemically will be competent to acquire the necessary initiation factors and have the potential to fire in that cell cycle (see Nomenclature). Origins that happen to be poor binding sites for ORC, for instance, may fail to form pre-RCs in some fraction of the cells and therefore not be competent to fire in those cells. Alternatively, complexes may have shorter half-lives at some origins than at others, again leading to situation that a proportion of the cells in the population may not be competent to fire that origin. Second, an origin that is fully competent to fire (i.e., all cells in the population have the biochemical requirements to fire that origin) may nevertheless fail to fire in some proportion of the cells if it is replicated passively in those cells-i.e., it happens to be replicated by an incoming fork before it actually fires. For example, S. cerevisiae ARS301 apparently can support plasmid maintenance with high fidelity, indicating that it has high competence, but it almost always is replicated passively in its native chromosomal context even though, as judged by footprinting, it does form a pre-RC (Newlon et al. 1993; Newlon and Theis 1993; Santocanale et al. 1999). In contrast, the rDNA ARS in $S$. cerevisiae appears to be an example of a low-competence origin - in its native context, it shows low efficiency $(<20 \%)$, and plasmids maintained by this ARS are lost at high frequency (Ivessa and Zakian 2002). The functional ACS in this instance shows a poor match to the consensus; replacing the native ACS with a better match to the consensus improves plasmid maintenance, suggesting again that the poor efficiency is a consequence of poor competence, which in turn arises from reduced ability to recruit ORC or other proteins (Miller et al. 1999).

\section{Temporal programs re-examined}

The poor firing success of origins in species ranging from $S$. pombe to humans has spurred a re-examination of the concept of a temporal program of replication (e.g., Rhind 2006) - if origin firing success is generally low, how biologically meaningful is a temporal program, and how might it be set up? One can consider models with different levels of determinism (Fig. 3). At one extreme is a strictly deterministic or "take a number" model. Rather like customers in some office being serviced according to the numbers on their tickets, origins in this model would fire in a set order when their "numbers" were called by some cellular clock (or after the previous numbers had been called); origins could perhaps miss a turn but never jump the queue and fire out of order. While we cannot at present rule out the possibility that there is an obligate order of replication or firing of select "timekeeper" or "master" origins, evidence overwhelmingly is against a deterministic, dependent model where the firing of each origin is contingent upon the successful firing of the previous origin in the sequence - were there such a dependency, any cell that failed to fire one origin would subsequently fail to fire its remaining unfired origins. In the population, one therefore would see a progressive decrease in origin firing efficiency at successively later times in S phase. In fact, while latefiring origins often tend to be less efficient than earlyfiring ones, there clearly are instances of efficient late origins (Friedman et al. 1996). Single-molecule analysis of replication both in $S$. cerevisiae and in $S$. pombe, despite some limitations in resolution and unambiguous identification of origins, also provide strong evidence against the existence of a deterministic order of origin firing (Patel et al. 2006; Czajkowsky et al. 2008).

At the other end of the spectrum is a model of strictly stochastic firing: origins compete equally for some critical initiation factor, rather like rail passengers rushing to find seats once the carriage doors have been thrown open. Successful firing is a matter of chance, and therefore there is no set order of origin firing. Stochastic firing had been proposed previously in the context of the rapid cycles of replication in early frog and fly embryos (Hyrien et al. 2003) and is compatible with the pattern of origin firings seen in those systems but clearly is not compatible with the 

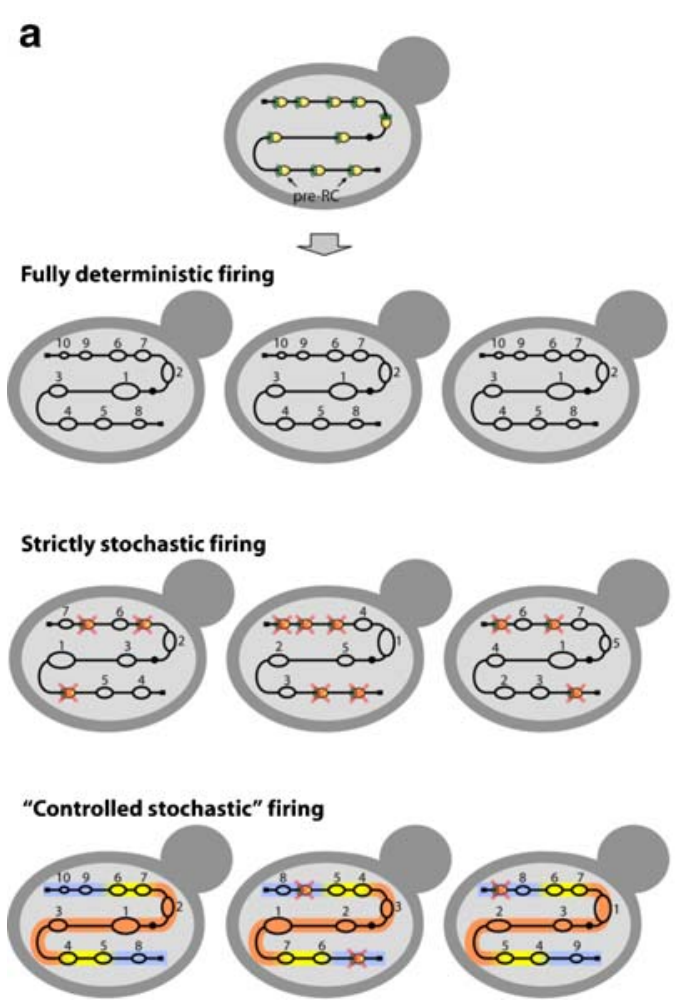

Fig. 3 The temporal program of replication. a Deterministic vs. stochastic models for origin firing time. In a strictly deterministic system, all cells in the population would fire their origins in the same temporal order (numbers above each bubble structure depicting the order of firing in that cell). In a strictly stochastic system, the firing sequence would be disordered. In a "controlled stochastic" or "fuzzy deterministic" system, chromatin or other forms of regulation could result in genomic regions showing differential access to critical initiation factors at different times in $\mathrm{S}$ phase, so that origins in different genomic zones could show different times of replication (red, yellow, and blue representing early-, mid-, and late-S zones; unfired pre-RCs indicated with red $x$ marks in this simplistic view), but stochastic processes would still act locally, precluding a set order of origin activation. b One prediction of such a

obvious temporal patterns of replication observed in species such as $S$. cerevisiae.

A middle-ground "controlled stochastic" or "fuzzy deterministic" model could account for the observed patterns of replication (discussed in more detail elsewhere in this volume). In this revised stochastic model, origins compete for limiting factors but there can be variation in the probability of firing different origins at different times in $\mathrm{S}$ phase. This model predicts a progressive decline in the probability of origin firing over S phase, which would appear not to be strictly the case in $S$. cerevisiae. For example, b

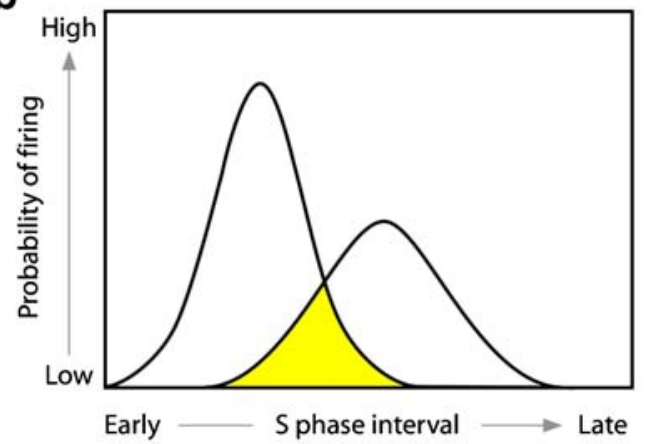

C

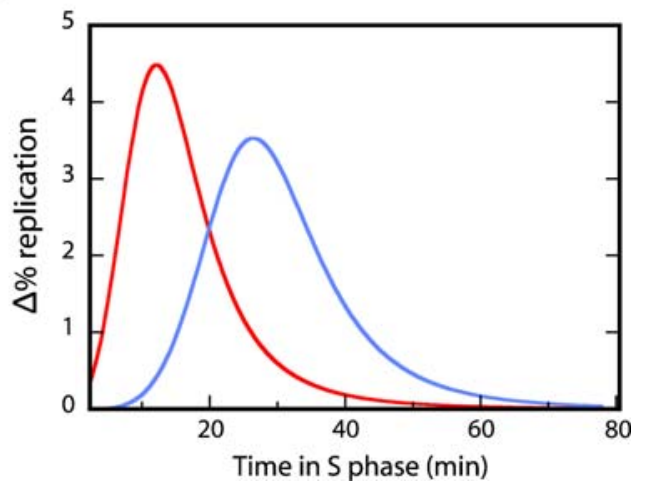

"controlled stochastic" model is that origins would show distributions of firing probabilities, with early and late origins showing distinct but potentially overlapping distributions (yellow), allowing for some disorder in the precise sequence of initiations but still resulting in a general temporal patterning of replication. c Firing probability distributions for $S$. cerevisiae ARS1413 on an early- (p13, red) vs. a late-replicating (pRightDup, blue) plasmid, inferred from the replication kinetic curves for these plasmids (Friedman et al. 1996; also see McCune et al. 2008 and Fig. 4). Because each plasmid had only one origin (ARS1413 in each case, with different amounts and arrangements of flanking genomic DNA included on the plasmid), the incremental change in percent replication $(\Delta \%$ replication) reflects the incremental origin firing occurring per 2-min time interval through $\mathrm{S}$ phase

different single-origin plasmids, where passive replication of the origins is not a concern, have been demonstrated to have distinct replication times and yet show little difference in loss rates (Ferguson et al. 1991; Ferguson and Fangman 1992; Friedman et al. 1996; Donaldson et al. 1998; Hoang et al. 2007). A second counterexample from $S$. cerevisiae is the replication behavior of a clb5 $\Delta$ strain (McCune et al. 2008). Clb5 and Clb6 are the two cyclins responsible for driving $\mathrm{S}$ phase in wild-type $S$. cerevisiae (Nasmyth 1996). In the absence of $\mathrm{Clb5}, \mathrm{S}$ phase begins on time, supported by Clb6- but because Clb6 
is degraded rapidly and is depleted before the end of $\mathrm{S}$ phase (Jackson et al. 2006), origin firing later in S phase is compromised (Donaldson et al. 1998). Replication profiling of clb5 $\Delta$ cells showed that the kinetics of replication of regions of the genome that normally would replicate early remained unchanged, whereas regions that normally would depend on latefiring replication were significantly delayed in replication (McCune et al. 2008). The observation that early origins show no diminution in activity in the mutant argues against a disordered stochastic model of origin timing: such a model would predict that all origins should be compromised (albeit to different extents) by a depletion of Cdk1 activity. Finally, the observation in animal cells of developmentally regulated changes in origin firing time argues for regulation of the probability of origin firing.

These issues can be addressed by proposing two additional features: one, that over the course of each $\mathrm{S}$ phase there is an increase in the intranuclear concentration of the hypothetical limiting factor by a combination of continued synthesis and release of the factor from origins that have already fired in that cell cycle (Rhind 2006); and two, that there can be regulated variation in origin firing probability, perhaps in the form of genomic zones or regions in which access to initiation factors can be restricted at particular times in $\mathrm{S}$ phase. In the clb5 study mentioned above, for instance, it was noted that different origins sampled at the same time within S phase showed different responses to the depletion of Cdk1 activity: whereas an "early" origin showed no diminution in firing activity, a "late" origin in those same cells showed decreased activity, arguing for differential ability of those origins to compete for successful firing within the same cells.

Thus, the reality appears to be best described as a mix of the previous two models (strictly deterministic and strictly stochastic): there is some regulation in the order of origin firing, but stochastic events do come into play perhaps at a more local level — rather like the zone-by-zone boarding of passenger airplanes. In this scenario, some origins would have their highest firing probability early in S phase while others would peak in firing probability later in $\mathrm{S}$, but overlaps between the peak periods (owing to stochastic processes) would result in some cell-to-cell variation in the precise order of origin activations (Fig. 3a, b). Some aspects of the $S$. cerevisiae replication program are indeed fully consistent with the idea that stochastic processes can yield ordered outcomes. For example, the amount of replication occurring in progressive S-phase intervals (the $\Delta \%$ replication) for an early- vs. a late-replicating derivative of a single-ARS plasmid shows distinct but overlapping distributions (Friedman et al. 1996; McCune et al. 2008). Because these are single-origin plasmids, the deduced temporal distribution of $\Delta \%$ replication reflects the probabilities of origin firing on each plasmid in different S-phase intervals. That the two distributions overlap and that the later origin shows a wider distribution (Fig. 3b, c) are both consistent with stochastic effects reflecting a competition for some limiting factor.

The extent to which stochastic processes predominate seems to depend on the species. In $S$. cerevisiae and in animal cells, replication time is re-established in every cell cycle (Raghuraman et al. 1997; Dimitrova and Gilbert 1999) and in animal cells in particular, replication timing appears to be set at the level of large, megabase-sized developmentally regulated domains (reviewed by Hiratani et al. 2009) - all arguing for regulated changes in state (chromatin and/or nuclear positioning, for instance) regulating changes in replication timing. The observation in $S$. cerevisiae that checkpoints play a role in regulating origin firing (Santocanale and Diffley 1998; Shirahige et al. 1998; Feng et al. 2006) further reinforces the notion that there is, at some level, cellular control over the time of origin activation in this yeast.

From single-molecule analysis of replication using stretched DNA, it appears that origin firing in $S$. pombe is much more stochastic and that successful firing of an origin in one cell cycle is not a predictor of successful firing in subsequent cell cycles (Patel et al. 2006). This conclusion is consistent with at least a qualitative assessment of 2-D gel results showing that, with a few exceptions, origins in $S$. pombe are very inefficient (Segurado et al. 2003). When it comes to the presence or absence of temporally regulated origin firing in $S$. pombe, however, four recent microarraybased surveys of replication, reaching somewhat different conclusions, have not really clarified matters. Two of these studies - one using accumulation of ssDNA as a signature of origin firing (Feng et al. 2006) and the other using incorporation of BrdU at sites of ORC binding as a measure of origin activity (Hayashi et al. 2007) - compared replication in wild type and a $c d s 1$ checkpoint mutant and found evidence for a 
substantial number of origins (up to 39\%) that fired in the checkpoint mutant but failed to fire in wild type in the presence of HU. The conclusion was that the putative origins that showed activity in $\mathrm{HU}$ in the checkpoint mutant but not in wild type corresponded to late-firing origins - analogous to the observation in S. cerevisiae that mutating RAD53, the functional equivalent of $c d s 1$, allows firing of origins that normally would be suppressed by the HU-triggered checkpoint (Santocanale and Diffley 1998; Feng et al. 2006). However, two other studies, both measuring copy number increase to monitor replication, came to a different conclusion. One study compared wild type and $\mathrm{rad} 3$, which acts upstream of $c d s 1^{+}$and therefore should give the same phenotype as a $c d s 1$ mutant (Heichinger et al. 2006); the other study compared wild type, $c d s 1$, and rad 3 strains and scored an origin as being checkpoint-suppressed only if a significant difference in the level of replication of that origin was found between wild type and both mutants (Mickle et al. 2007). Both studies concluded that only a very small number $(\leq 3 \%)$ of origins showed evidence of firing in checkpoint-deficient compared to wild-type cellssuggesting that late-firing origins, if they exist, are a rarity in $S$. pombe.

One possible explanation behind these apparently contradictory results is that $\mathrm{rad} 3$ and $c d s 1$, although they are in the same checkpoint pathway, actually have distinct phenotypes when it comes to origin activation. In fact, Mickle et al. (2007) found $c d s 1$ specific differences in apparent origin activity, although they were justifiably cautious in noting that such differences could simply be noise in the data. It is noteworthy that a rad53 and a mecl mutant in $S$. cerevisiae have also recently been found to differ in the extent of replication in the presence of HU and in their ability to recover from the drug treatment, although these two checkpoint genes are thought to function in the same pathway (Feng et al. 2009). An alternative idea (Heichinger et al. 2006; Mickle et al. 2007 ) is that the amount of ssDNA or BrdU incorporation at origins was just higher in $c d s 1$ cells than in wild type and that some origins that were scored as being active in $c d s 1$ were scored as inactive in wild type just because the signal (BrdU incorporation or ssDNA) at those origins fell below the chosen cutoff. The recent observation that a heterochromatic protein is involved in promoting early activation of pericentric origins suggests that the activation time of some origins, at least, is regulated (Hayashi et al. 2009).

Finally, in animal cells, the very concept of origins has become blurred. The observation that initiation can occur at multiple sites within a relatively small region has led to the idea that an origin is not a specific DNA sequence that coincides with the site where DNA synthesis is initiated but rather that an origin is a small region consisting of a cluster of initiation sites (Hamlin et al. 2008). Within the cluster, initiation again is stochastic, although there clearly is regulation of replication on a global level (Kitsberg et al. 1993; Norio et al. 2005; Desprat et al. 2009).

The elephant in the room, of course, is the question of what the function might be of temporal programs of replication. In a purely stochastic system, there could well be no specific function. However, the observation of conserved early replication of centromeres in $S$. cerevisiae and $S$. pombe argues for some functional consequence of temporally distinct replication times (McCarroll and Fangman 1988; Raghuraman et al. 2001; Hayashi et al. 2009). Recently, we have found that early replication of centromeres, coordinated in a checkpoint-dependent mechanism with the segregation apparatus in S. cerevisiae, is essential for maintaining viability in the face of replication stress: rad53 mutants exposed to $\mathrm{HU}$ fail to replicate their centromeres in time to prevent premature attachment of the unreplicated chromosomes to the spindle, resulting in random segregation of their unreplicated chromosomes and concomitant aneuploidy upon removal of HU (Feng et al. 2009).

In animal cells, too, the observation of developmentally regulated changes in replication time has long been taken as evidence of a functional role for temporal patterning of replication, especially given the correlation of transcriptional potential with early replication. As discussed above, recent studies on replication time in relation to transcriptional activity in a variety of animal cells have strengthened the view that replication may be an upstream indicator or regulator of transcriptional activity (e.g., Goren et al. 2008; Desprat et al. 2009).

One possibility that cannot at present be dismissed is that we are looking at the wrong places: with few exceptions (e.g., Collins and Newlon 1994; Heichinger et al. 2006), studies on replication have been done 
almost exclusively on mitotically cycling cells. It is possible that temporal replication patterns that we observe in such cells are an echo of a pattern that is really needed in other situations, e.g., in premeiotic $\mathrm{S}$ phase or in early development (or spore germination in yeast). The early replication cycles observed in Xenopus systems and amplification of specific genes in insects probably are the best proxies for investigations into replication during early development at this point, although recent studies of replication in stem cells before and after differentiation show tremendous promise (Hiratani et al. 2008). Ultimately, it will require experimental manipulation of replication time and examination of potential phenotypes that might result, to really understand the possible roles for the replication program. Yeasts may again lead the way, given the relative ease of performing competitive growth and experimental evolution analyses in these model organisms.

\section{Mathematical modeling of replication}

Whatever the explanation for the observed patterns of replication, settling the issue of the underlying mechanism will need more work. But where does that leave us in understanding how temporal patterns might arise? Specification of origin choice and firing time is a complex process, and our intuition can break down when predicting outcomes of particular scenarios or sets of parameters (e.g., predicting replication profiles of chromosomes where the firing time or firing competence of particular origins has been altered). Many researchers in recent years have come to the realization that mathematical modeling of replication would be a powerful complement to our understanding of the underlying molecular processes. At the very least, such exercises aid our intuition and can alert us about incorrect assumptions - e.g., we can begin with a set of assumptions (origin locations, firing times, etc.) and ask whether, given that set of assumptions, the predicted replication profiles match the actual observations. If set up appropriately, modeling can also be used to refine hypotheses and to fit parameters to experimental data and thereby obtain estimates of, for example, origin competence or firing time.

Thus, for instance, modeling a scenario of inefficient origins (potential origins vastly outnumbering the actual number of origins, which would show cellto-cell variation) and a limiting concentration of a required initiation factor has helped clarify the thinking about the nature of the replication program in $S$. pombe and has set up specific hypotheses to test (e.g., the role of a limiting factor; Rhind 2006; Patel et al. 2008). Such modeling has led to the idea that recycling and import of a limiting factor would alleviate the potential problem of large unreplicated regions remaining behind in the genome if origins were inefficient and chosen at random from a pool of potential sites; the prediction of such a model is that the firing rate or spatiotemporal density of initiations (initiations per kilobase per unit time) would increase over S phase so that the probability of unreplicated regions would be low (Rhind 2006). Contrary to these expectations, however, a recent examination of replication profiling in several species ( $S$. cerevisiae, S. pombe, Drosophila melanogaster, Xenopus laevis, and Homo sapiens) would appear to show that while the spatiotemporal density of initiations increases for the first part of S phase, it subsequently decreases in all species examined (Goldar et al. 2009); a conclusion supported from data in a separate study in $S$. cerevisiae (McCune et al. 2008). Mathematical modeling again suggested a possible resolution to this apparent paradox: that if initiations were coupled to the density of replication forks (increased fork density promoting association between origins and a limiting factor), then as forks merged and were resolved as $\mathrm{S}$ phase progresses, the probability of initiations would also decrease (Goldar et al. 2008).

Of course, modeling only suggests possibilities that are consistent with the experimental results: for example, modeling can suggest that fork density may account for the density of initiations, but does not in any way prove that it is responsible for the observed results. Given the potentially large constellation of variables involved, multiple scenarios could potentially give very similar outcomes; modeling and experimental tests of predictions from the modeling should ideally be used as steps in an iterative process. It seems likely that, at least initially, modeling will be most fruitful in simple systems where more of the parameters are known. As a relatively simple example, modeling of replication in S. cerevisiae, where origin locations and temporal replication profiles are known, can yield numerical predictions of efficiency and of competence for specific origins; the predicted 
efficiencies can then be tested empirically. Such modeling can also set up predictions of what the replication profiles would be for mutants in which specific origins have been eliminated by mutation. In any event, the recent surge in interest in modeling (Cadoret et al. 2008; Lygeros et al. 2008; Spiesser et al. 2009; and Yang et al. 2009 are but a few examples) bodes well for the prospects of detailed molecular models to test in the future.

\section{Regulating origin firing time}

The nonrandom clustering of origins with similar firing times (Yabuki et al. 2002; McCune et al. 2008) argues for temporal control over replication at the level of genomic domains. By what means might cells influence the time of origin activation? Modulation of chromatin structure is an obvious means of regulating replication time, with subnuclear localization being a runner-up. In S. cerevisiae, as demonstrated by origin transplantation experiments, origin firing time is influenced by the chromosomal context (Ferguson and Fangman 1992; Friedman et al. 1996) - i.e., sequences outside of the origin itself act to delay the firing of the origin. Two such examples have been examined in some detail (Fig. 4). In one instance, proximity to telomeres was found to delay origin activation through a SIR3-dependent process-indi- cating that chromatin structure must be involved (Ferguson and Fangman 1992; Stevenson and Gottschling 1999). Subsequently, cells deficient in the telomere binding protein $\mathrm{Ku}$ were also found to show advanced firing of subtelomeric origins (Cosgrove et al. 2002), again consistent with the idea that chromatin structure influences origin activation time. In the other example of a demonstrated position effect on origin activation in $S$. cerevisiae, small regions of chromosome XIV were found to delay firing of adjacent origins (Friedman et al. 1996). Little is known about the underlying mechanism in this instance, except that it appears to be independent of the telomeric chromatin mechanism. Nevertheless, the fact that relatively small and defined DNA sequences can affect origin activation keeps alive the hope that it will be possible to uncover at least some of the molecular mechanisms governing origin timing.

If chromatin structure is involved, histone modification is likely to play a role. Indeed, loss of the Rpd3 histone deacetylase in $S$. cerevisiae results in precocious replication of many usually late-S-activated origins (Vogelauer et al. 2002; Knott et al. 2009). Many of the origins that show altered firing time are closely linked to genes that show altered levels of transcription, underscoring again the potential link between gene expression and replication timingalthough again, without having verified the actual firing times, it remains possible that the less con-

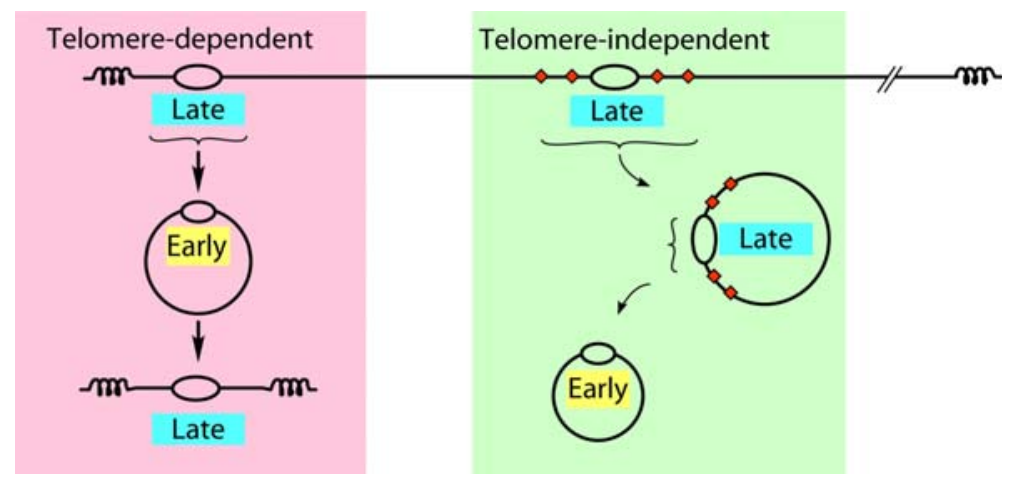

Fig. 4 Cis-acting determinants of origin firing time in $S$. cerevisiae. Proximity to telomeres results in delayed firing of origins; chromatin is known to be a regulator of origin firing in this instance although the details of chromatin spreading from the telomere over 25+-kb distances are not well understood (Ferguson et al. 1991; Stevenson and Gottschling 1999; Cosgrove et al. 2002). Internal late-firing origins presumably are regulated by nontelomeric sequences proximal to the origins. One example is the cluster of late origins on $S$. cerevisiae chromosome XIV, where several sequences adjacent to the origins are known to act additively to delay origin firing time, as determined by monitoring the replication times of plasmids containing these origins along with different amounts of flanking sequence (Friedman et al. 1996). The precise nature of the timing determinants in this example is not known, but it has been shown that targeting a histone acetylase to one of the origins results in an advancement in its firing time (Vogelauer et al. 2002) 
densed chromatin in the rpd3 mutant causes increased origin firing efficiency rather than advanced firing time (Knott et al. 2009).

Histone acetylation can affect replication timing in animal cells also. The human B-globin gene cluster is replicated early in $\mathrm{S}$ phase in erythroid cells and late in nonerythroid cells; targeting a deactylase to the region (carried on a BAC introduced into mice) delayed its replication in erythroid cells while targeting a histone acetylase advanced its replication time in lymphoid cells (Goren et al. 2008). Although not a perfect system-human DNA examined in mouse cells and the switches in replication time were only partial - the results underscore the potential role of chromatin structure in replication.

A different (but not mutually exclusive) mechanism of replication timing could involve subnuclear localization of sequences. In $S$. cerevisiae, late origins spend relatively more time near the nuclear periphery than do early origins (Heun et al. 2001), although peripheral positioning is not necessary for late replication of telomeres (Hiraga et al. 2006). In animal cells, too, replication timing changes can be accompanied by changes in subnuclear positioning of the relevant sequences (Hiratani et al. 2008). Further exploration of this idea will have to await detailed spatial maps of the genome.

What exactly is the proposed limiting factor that is so central to the observed pattern of origin firing? In S. cerevisiae, Cdk1 activity can be made limiting for origin firing (by eliminating the S-phase cyclin Clb5, for instance)-but doing so does not delay origin firing as one might expect if $\mathrm{Cdk} 1$ were central to setting the pace of origin activations; instead, the residual firing that does occur still happens at the same time in S phase as in wild-type cells (McCune et al. 2008). Furthermore, elevated levels of Clb6 merely restored the normal pattern of replication in a clb5 mutant and did not cause precocious origin activation, indicating again that $\mathrm{Cdk} 1$ is not itself responsible for establishing the temporal pattern of origin activation (McCune et al. 2008). In S. pombe, there is evidence that Dbf4-dependent kinase (DDK) could be limiting for origin activation (Patel et al. 2008). If stochastic processes do predominate in $S$. pombe as mentioned previously, then DDK concentrations could also be involved in setting the pace of origin activations and thereby affect the replication program. Recent investigations into the targets of Cdk1 and DDK in $S$. cerevisiae hold out the promise that, with a better understanding of the roles of these essential kinases, we will be in a better position to uncover potential candidates for factors that limit the pace of origin activation.

S. cerevisiae cells entering $\mathrm{S}$ phase in the presence of HU delay the activation of all unfired origins in proportion to the slowdown of ongoing DNA synthesis, resulting in an S phase apparently moving in slow motion (Alvino et al. 2007). This regulation of $\mathrm{S}$ phase in response to HU-induced stress requires activation of the Rad53 checkpoint kinase, which is known to have among its downstream targets proteins required for origin activation such as Dbf4 (Santocanale and Diffley 1998; Shirahige et al. 1998). If a limiting factor does increase in availability as $\mathrm{S}$ phase progresses, then this factor must be regulated by Rad53; otherwise, the concentrations of the factor might be expected to exceed normal levels (due to the prolonged $\mathrm{S}$ phase in $\mathrm{HU}$ ) and thereby cause an elevation in origin efficiencywhich has not been observed. In fact, the "slow motion" nature of the HU S phase argues for some coupling between existing forks and future initiations as discussed by Rhind (2008).

\section{Evolution of origin specificity and the temporal program}

How might the different systems of replication (tightly specified origin locations and some specification of firing time, vs. stochastic origin location and perhaps little or no specification of firing time) have evolved? It is perhaps reasonable to speculate that the sizes of intergenic regions might have something to do with the sequence specificity of ORC binding. Transcription can disrupt origin function and may therefore drive selection of origin locations to intergenic regions (Brewer 1988; Snyder et al. 1988; Brewer 1994); increased binding specificity of ORC could then ensure that origin locations are maintained in the small intergenic regions. The availability of larger intergenic regions would relax the necessity for specificity and thereby increase the number of potential origins. But with the large number of potential origins could come the necessity to limit initiations to a subset of them-either because initiations potentially are destabilizing to the genome 
(Di Rienzi et al. 2009; Gordon et al. 2009) or because other factors, such as replication fork components, may become limiting. Chromatin or other modifiers could then act on the origins (either individually or as residents of domains) to impose temporally staggered firing patterns.

The ballooning compendium of yeast species with sequenced genomes presents the prospects of actually testing some of these ideas. Among yeasts, we have $S$. cerevisiae as an example of a yeast with fairly wellspecified origin locations and $S$. pombe at the other extreme as an example of a yeast with almost no sequence specificity at its origins. The wealth of sequence information presents a unique opportunity for a detailed comparative genomic analysis of replication-to ask how origin sequences have evolved and how that has affected the evolution of the temporal program of replication. In our laboratory, we have embarked on just such an odyssey. The next decade promises to be as exciting as the previous one.

Acknowledgments We would like to thank Conrad Nieduszynski and the other authors in this volume for their insights and discussions. This work was supported by the National Institute of General Medical Sciences grant 18926 to M. K. R. and B. J. B.

\section{References}

Alvino GM, Collingwood D, Murphy JM et al (2007) Replication in hydroxyurea: it's a matter of time. Mol Cell Biol 27:6396-6406

Bell SP, Dutta A (2002) DNA replication in eukaryotic cells. Annu Rev Biochem 71:333-374

Bell SP, Stillman B (1992) ATP-dependent recognition of eukaryotic origins of DNA replication by a multiprotein complex. Nature 357:128-134

Blow JJ, Laskey RA (1988) A role for the nuclear envelope in controlling DNA replication within the cell cycle. Nature 332:546-548

Brewer BJ (1988) When polymerases collide: replication and the transcriptional organization of the E. coli chromosome. Cell 53:679-686

Brewer BJ (1994) Intergenic DNA and the sequence requirements for replication initiation in eukaryotes. Curr Opin Genet Dev 4:196-202

Brewer BJ, Fangman WL (1987) The localization of replication origins on ARS plasmids in $S$. cerevisiae. Cell 51:463-471

Cadoret JC, Meisch F, Hassan-Zadeh V et al (2008) Genomewide studies highlight indirect links between human replication origins and gene regulation. Proc Natl Acad Sci U S A 105:15837-15842
Clyne RK, Kelly TJ (1995) Genetic analysis of an ARS element from the fission yeast Schizosaccharomyces pombe. EMBO J 14:6348-6357

Collins I, Newlon CS (1994) Chromosomal DNA replication initiates at the same origins in meiosis and mitosis. Mol Cell Biol 14:3524-3534

Cosgrove AJ, Nieduszynski CA, Donaldson AD (2002) Ku complex controls the replication time of DNA in telomere regions. Genes Dev 16:2485-2490

Czajkowsky DM, Liu J, Hamlin JL, Shao Z (2008) DNA combing reveals intrinsic temporal disorder in the replication of yeast chromosome VI. J Mol Biol $375: 12-19$

Desprat R, Thierry-Mieg D, Lailler N et al. (2009) Predictable dynamic program of timing of DNA replication in human cells. Genome Res (in press)

Di Rienzi S, Collingwood D, Raghuraman MK, Brewer BJ (2009) Fragile genomic sites are associated with origins of replication. Genome Anal Evolut 1:350-363

Diffley JF, Cocker JH (1992) Protein-DNA interactions at a yeast replication origin. Nature 357:169-172

Dimitrova DS, Gilbert DM (1999) The spatial position and replication timing of chromosomal domains are both established in early G1 phase. Mol Cell 4:983-993

Donaldson AD (2005) Shaping time: chromatin structure and the DNA replication programme. Trends Genet 21:444-449

Donaldson AD, Raghuraman MK, Friedman KL et al (1998) CLB5-dependent activation of late replication origins in $S$. cerevisiae. Mol Cell 2:173-182

Fangman WL, Brewer BJ (1992) A question of time: replication origins of eukaryotic chromosomes. Cell 71:363-366

Feng W, Collingwood D, Boeck ME et al (2006) Genomic mapping of single-stranded DNA in hydroxyureachallenged yeasts identifies origins of replication. Nat Cell Biol 8:148-155

Feng W, Raghuraman MK, Brewer BJ (2007) Mapping yeast origins of replication via single-stranded DNA detection. Methods 41:151-157

Feng W, Bachant J, Collingwood D, Raghuraman MK, Brewer BJ (2009) Centromere replication timing determines different forms of genomic instability in Saccharomyces cerevisiae checkpoint mutants during replication stress. Genetics (in press)

Ferguson BM, Fangman WL (1992) A position effect on the time of replication origin activation in yeast. Cell 68:333-339

Ferguson BM, Brewer BJ, Reynolds AE, Fangman WL (1991) A yeast origin of replication is activated late in $\mathrm{S}$ phase. Cell 65:507-515

Friedman KL, Brewer BJ (1995) Analysis of replication intermediates by two-dimensional agarose gel electrophoresis. Methods Enz 262:613-627

Friedman KL, Diller JD, Ferguson BM et al (1996) Multiple determinants controlling activation of yeast replication origins late in S phase. Genes Dev 10:1595-1607

Friedman KL, Brewer BJ, Fangman WL (1997) Replication profile of Saccharomyces cerevisiae chromosome VI. Genes Cells 2:667-678

Gartler SM, Goldstein L, Tyler-Freer SE, Hansen RS (1999) The timing of XIST replication: dominance of the domain. Hum Mol Genet 8:1085-1089 
Goldar A, Labit H, Marheineke K, Hyrien O (2008) A dynamic stochastic model for DNA replication initiation in early embryos. PLoS ONE 3:e2919

Goldar A, Marsolier-Kergoat MC, Hyrien O (2009) Universal temporal profile of replication origin activation in eukaryotes. PLoS ONE 4:e5899

Gordon JL, Byrne KP, Wolfe KH (2009) Additions, losses, and rearrangements on the evolutionary route from a reconstructed ancestor to the modern Saccharomyces cerevisiae genome. PLoS Genet 5:e1000485

Goren A, Tabib A, Hecht M, Cedar H (2008) DNA replication timing of the human beta-globin domain is controlled by histone modification at the origin. Genes Dev 22:1319-1324

Hamlin JL, Mesner LD, Lar O et al (2008) A revisionist replicon model for higher eukaryotic genomes. J Cell Biochem 105:321-329

Hansen RS, Canfield TK, Gartler SM (1995) Reverse replication timing for the XIST gene in human fibroblasts. Hum Mol Genet 4:813-820

Hayashi M, Katou Y, Itoh T et al (2007) Genome-wide localization of pre-RC sites and identification of replication origins in fission yeast. Embo J 26:1327-1339

Hayashi MT, Takahashi TS, Nakagawa T, Nakayama J, Masukata H (2009) The heterochromatin protein Swi6/ HP1 activates replication origins at the pericentromeric region and silent mating-type locus. Nat Cell Biol 11:357-362

Heichinger C, Penkett CJ, Bahler J, Nurse P (2006) Genomewide characterization of fission yeast DNA replication origins. EMBO J 25:5171-5179

Heinzel SS, Krysan PJ, Tran CT, Calos MP (1991) Autonomous DNA replication in human cells is affected by the size and the source of the DNA. Mol Cell Biol 11:2263-2272

Heun P, Laroche T, Raghuraman MK, Gasser SM (2001) The positioning and dynamics of origins of replication in the budding yeast nucleus. J Cell Biol 152:385-400

Hiraga S, Robertson ED, Donaldson AD (2006) The Ctf18 RFC-like complex positions yeast telomeres but does not specify their replication time. EMBO J 25:1505-1514

Hiratani I, Ryba T, Itoh M et al (2008) Global reorganization of replication domains during embryonic stem cell differentiation. PLoS Biol 6:e245

Hiratani I, Takebayashi S, Lu J, Gilbert DM (2009) Replication timing and transcriptional control: beyond cause and effectpart II. Curr Opin Genet Dev 19:142-149

Hoang ML, Leon RP, Pessoa-Brandao L et al (2007) Structural changes in $\mathrm{Mcm} 5$ protein bypass Cdc7-Dbf4 function and reduce replication origin efficiency in $S$. cerevisiae. Mol Cell Biol 27:7594-7602

Hyrien O, Marheineke K, Goldar A (2003) Paradoxes of eukaryotic DNA replication: MCM proteins and the random completion problem. Bioessays 25:116-125

Irene C, Maciariello C, Cioci F et al (2004) Identification of the sequences required for chromosomal replicator function in Kluyveromyces lactis. Mol Microbiol 51:1413-1423

Ivessa AS, Zakian VA (2002) To fire or not to fire: origin activation in Saccharomyces cerevisiae ribosomal DNA. Genes Dev 16:2459-2464
Jackson LP, Reed SI, Haase SB (2006) Distinct mechanisms control the stability of the related S-phase cyclins Clb5 and Clb6. Mol Cell Biol 26:2456-2466

Kim SM, Huberman JA (2001) Regulation of replication timing in fission yeast. Embo J 20:6115-6126

Kitsberg D, Selig S, Keshet I, Cedar H (1993) Replication structure of the human beta-globin gene domain. Nature 366:588-590

Knott SR, Viggiani CJ, Tavare S, Aparicio OM (2009) Genome-wide replication profiles indicate an expansive role for $\operatorname{Rpd} 3 \mathrm{~L}$ in regulating replication initiation timing or efficiency, and reveal genomic loci of Rpd3 function in Saccharomyces cerevisiae. Genes Dev 23:1077-1090

Lengronne A, Pasero P, Bensimon A, Schwob E (2001) Monitoring $\mathrm{S}$ phase progression globally and locally using BrdU incorporation in $\mathrm{TK}(+)$ yeast strains. Nucleic Acids Res 29:1433-1442

Lucas I, Palakodeti A, Jiang Y et al (2007) High-throughput mapping of origins of replication in human cells. EMBO Rep 8:770-777

Lygeros J, Koutroumpas K, Dimopoulos S et al (2008) Stochastic hybrid modeling of DNA replication across a complete genome. Proc Natl Acad Sci U S A 105:1229512300

Marahrens Y, Stillman B (1992) A yeast chromosomal origin of DNA replication defined by multiple functional elements. Science 255:817-823

McCarroll RM, Fangman WL (1988) Time of replication of yeast centromeres and telomeres. Cell 54:505-513

McCune HJ, Danielson LS, Alvino GM et al (2008) The temporal program of chromosome replication: genomewide replication in clb5 \{Delta\} Saccharomyces cerevisiae. Genetics 180:1833-1847

Meselson M, Stahl FW (1958) The replication of DNA in Escherichia coli. Proc Natl Acad Sci U S A 44:671-682

Mickle KL, Ramanathan S, Rosebrock A et al (2007) Checkpoint independence of most DNA replication origins in fission yeast. BMC Mol Biol 8:112

Miller CA, Umek RM, Kowalski D (1999) The inefficient replication origin from yeast ribosomal DNA is naturally impaired in the ARS consensus sequence and in DNA unwinding. Nucleic Acids Res 27:3921-3930

Nasmyth K (1996) At the heart of the budding yeast cell cycle. Trends Genet 12:405-412

Newlon CS, Theis JF (1993) The structure and function of yeast ARS elements. Curr Opin Genet Dev 3:752-758

Newlon CS, Collins I, Dershowitz A et al (1993) Analysis of replication origin function on chromosome III of Saccharomyces cerevisiae. Cold Spring Harb Symp Quant B 58:415-423

Norio P, Kosiyatrakul S, Yang Q et al (2005) Progressive activation of DNA replication initiation in large domains of the immunoglobulin heavy chain locus during B cell development. Mol Cell 20:575-587

Okuno Y, Okazaki T, Masukata H (1997) Identification of a predominant replication origin in fission yeast. Nucleic Acids Res 25:530-537

Patel PK, Arcangioli B, Baker SP, Bensimon A, Rhind N (2006) DNA replication origins fire stochastically in fission yeast. Mol Biol Cell 17:308-316 
Patel PK, Kommajosyula N, Rosebrock A et al (2008) The Hsk1(Cdc7) replication kinase regulates origin efficiency. Mol Biol Cell 19:5550-5558

Raghuraman MK, Brewer BJ, Fangman WL (1997) Cell cycledependent establishment of a late replication program. Science 276:806-809

Raghuraman MK, Winzeler EA, Collingwood D et al (2001) Replication dynamics of the yeast genome. Science 294:115-121

Rao PN, Johnson RT (1970) Mammalian cell fusion: studies on the regulation of DNA synthesis. Nature 225:159-164

Rhind N (2006) DNA replication timing: random thoughts about origin firing. Nat Cell Biol 8:1313-1316

Rhind N (2008) An intrinsic checkpoint model for regulation of replication origins. Cell Cycle 7:2619-2620

Santocanale C, Diffley JF (1996) ORC- and Cdc6-dependent complexes at active and inactive chromosomal replication origins in Saccharomyces cerevisiae. Embo J 15:6671-6679

Santocanale C, Diffley JF (1998) A Mec1- and Rad53-dependent checkpoint controls late-firing origins of DNA replication. Nature 395:615-618

Santocanale C, Sharma K, Diffley JF (1999) Activation of dormant origins of DNA replication in budding yeast. Genes Dev 13:2360-2364

Schübeler D, Scalzo D, Kooperberg C et al (2002) Genome-wide DNA replication profile for Drosophila melanogaster: a link between transcription and replication timing. Nat Genet 32:438-442

Segurado M, de Luis A, Antequera F (2003) Genome-wide distribution of DNA replication origins at A+T-rich islands in Schizosaccharomyces pombe. EMBO Rep 4:1048-1053

Shirahige K, Hori Y, Shiraishi K et al (1998) Regulation of DNA-replication origins during cell-cycle progression. Nature 395:618-621

Snyder M, Sapolsky RJ, Davis RW (1988) Transcription interferes with elements important for chromosome maintenance in Saccharomyces cerevisiae. Mol Cell Biol 8:2184-2194

Spiesser TW, Klipp E, Barberis M (2009) A model for the spatiotemporal organization of DNA replication in Saccharomyces cerevisiae. Mol Genet Genomics 282:25-35

Stevenson JB, Gottschling DE (1999) Telomeric chromatin modulates replication timing near chromosome ends. Genes Dev 13:146-151
Stinchcomb DT, Thomas M, Kelly J, Selker E, Davis RW (1980) Eukaryotic DNA segments capable of autonomous replication in yeast. Proc Natl Acad Sci U S A 77:4559-4563

Taylor JH (1960) Asynchronous duplication of chromosomes incultured cells of Chinese hamster. J Biophysic Biochem Cytol 7:455-463

Theis JF, Newlon CS (1997) The ARS309 chromosomal replicator of Saccharomyces cerevisiae depends on an exceptional ARS consensus sequence. Proc Natl Acad Sci U S A 94:10786-10791

Theis JF, Newlon CS (2001) Two compound replication origins in Saccharomyces cerevisiae contain redundant origin recognition complex binding sites. Mol Cell Biol 21:2790-2801

van Brabant AJ, Hunt SY, Fangman WL, Brewer BJ (1998) Identifying sites of replication initiation in yeast chromosomes: looking for origins in all the right places. Electrophoresis 19:1239-1246

Vogelauer M, Rubbi L, Lucas I, Brewer BJ, Grunstein M (2002) Histone acetylation regulates the time of replication origin firing. Mol Cell 10:1223-1233

Woodfine K, Fiegler H, Beare DM et al (2004) Replication timing of the human genome. Hum Mol Genet 13:191-202

Wyrick JJ, Aparicio JG, Chen T et al (2001) Genome-wide distribution of ORC and MCM proteins in S. cerevisiae: high-resolution mapping of replication origins. Science 294:2357-2360

Xu W, Aparicio JG, Aparicio OM, Tavare S (2006) Genomewide mapping of ORC and Mcm2p binding sites on tiling arrays and identification of essential ARS consensus sequences in $S$. cerevisiae. BMC Genomics 7:276

Yabuki N, Terashima H, Kitada K (2002) Mapping of early firing origins on a replication profile of budding yeast. Genes Cells 7:781-789

Yamashita M, Hori Y, Shinomiya T et al (1997) The efficiency and timing of initiation of replication of multiple replicons of Saccharomyces cerevisiae chromosome VI. Genes Cells 2:655-665

Yang SC, Gauthier MG, Bechhoefer J (2009) Computational methods to study kinetics of DNA replication. Methods Mol Biol 521:555-573

Zannis-Hadjopoulos M, Sibani S, Price GB (2004) Eucaryotic replication origin binding proteins. Front Biosci 9:2133-2143 